\title{
TRATAMIENTO SUPERFICIAL CON NANOPARTÍCULAS FUNCIONALIZADAS (NF) PARA LA CONSERVACIÓN DE MAMPOSTERÍA ORNAMENTAL DE ARCILLA COCIDA
}

\author{
M. NERI-ÁLVAREZ, G. FAJARDO-SAN MIGUEL, D. CRUZ-MORENO \\ Universidad Autónoma de Nuevo León, Facultad de Ingeniería Civil, Av. Universidad S/N, Ciudad Universitaria, San \\ Nicolás de los Garza, Nuevo León C.P. 66455, México.
}

\section{RESUMEN}

El agua es el principal factor de degradación en la mampostería ornamental causada por la acción combinada y sinérgica, de los agentes biológicos, físicos y químicos. Debido a esto, el desarrollo de tratamientos de conservación se ha convertido en un tema de interés. En este trabajo, se evaluó un tratamiento superficial con nanopartículas funcionalizadas base silicio (NF) en tejas de arcilla cocida. La dosificación de las NF fue de $0.1 \%$ con respecto al volumen de agua necesaria para recubrir la superficie del material. Las muestras fueron expuestas en un ambiente exterior por 12 meses. Los resultados obtenidos mostraron que el producto sintetizado aportó propiedades hidrófobas en la superficie sin cambio significativo en el color del material.

Palabras clave: Nanomateriales; Tratamiento superficial; conservación; hidrofóbico; mampostería.

\section{ABSTRACT}

Water is the main degradation factor in ornamental masonry caused by the combined and synergistic action of biological, physical and chemical agents. The development of conservation treatments has become a topic of interest due to this. A surface treatment with silicon based functionalized nanoparticles (NF) in baked clay tiles was evaluated. The dosage of NF was $0.1 \%$ with respect to the volume of water needed to coat the surface of the material. The samples were exposed in an external environment for a period of one year. The results indicate that the product synthesized provides hydrophobic properties and does not change the color significantly.

Keywords: Nanomaterials; Surface treatment; conservation; hydrophobic; masonry.

\section{RESUMO}

A água é o principal fator de degradação da alvenaria ornamental causada pela ação combinada e sinérgica de agentes biológicos, físicos e químicos. Devido a isso, o desenvolvimento de tratamentos de conservação tornou-se um tópico de interesse. Neste trabalho, um tratamento de superfície com nanopartículas funcionalizadas baseadas em silício (NF) em telhas de argila cozidas foi avaliado. A dosagem do NF foi de $0,1 \%$ em relação ao volume de água necessário para revestir a superfície do material. As amostras foram expostas em ambiente externo por um período de um ano. Os resultados indicam que o produto é sintetizado.

Palavras-chave: Nanomateriais; Tratamento de superfície; conservação; hidrofóbico; alvenaria 


\section{INTRODUCCIÓN}

En México el uso de mampostería de arcilla cocida es empleada en acabados arquitectónicos, nuevas edificaciones, al igual que en monumentos de interés histórico. Sin embargo, el uso de tratamientos preventivos no es muy común en estos elementos. Según el ACI 116R-90 la mampostería es un sistema constructivo a base de unidades preformadas o moldeadas generalmente lo suficientemente pequeñas para ser manejadas por una sola persona y que están hechas de piedra, ladrillo o azulejo cocido, concreto, vidrio adobe o materiales similares. El agua es el principal factor de degradación en la mampostería ornamental ya que sirve como medio de transporte para los agentes biológicos, físicos y químicos, que actúan en acción combinada y sinérgica. Existen diferentes tipos de deterioro:

a) Deterioro por agentes Biológicos: Un sustrato constantemente húmedo se convierte en un hábitat favorable para la proliferación de organismos como musgos, líquenes, algas y mohos. Estos microorganismos en el exterior pueden deteriorar los materiales de construcción.

b) Deterioro por Factores Térmicos: El agua es considerada un conductor térmico que se acumula y humedece las unidades de mampostería, aumentando la dispersión térmica.

c) Deterioro Químico: Un exceso de agua incorpora sales, y recoge también los agentes químicos agresivos presentes en la atmósfera (como el anhídrido sulfuroso o carbónico). La interacción de la sustancia disuelta con los ligantes calcáreos, la cal y el carbonato de calcio presentes, los hace más solubles y propicia un rápido deterioro de la consistencia de la mampostería (Shaquihuanga-Ayala, D., 2014).

Todos estos tipos de deterioro se pueden prevenir o retardar con tratamientos superficiales aplicados poco después de ser colocados. El desarrollo de tratamientos de conservación se ha convertido en un tema de interés para la industria de la construcción, combatiendo diversos agentes de deterioro. En la actualidad, se han incorporado a los productos comerciales con materiales basados en nanopartículas (NPs), ya que desarrollan propiedades hidrófobas y autolimpiantes que promueven características notables frente a los agentes ambientales en comparación con los materiales de recubrimiento convencionales, mostrando un alto rendimiento y alta eficiencia energética (Boostani, et. al 2016). Algunos ejemplos de NPs incluyen de $\mathrm{Ca}(\mathrm{OH})_{2}$ y $\mathrm{SiO}_{2}$ para consolidación, NPs hidrofóbicas de $\mathrm{SiO}_{2}$, NPs de $\mathrm{TiO}_{2}$ para autolimpieza y efecto biocida (Ivask et al., 2012), así como NPs metálicas biocidas (Carrillo-González et al., 2016). La aplicación de productos obtenidos por una ruta sol-gel basados en $\mathrm{SiO}_{2}$ es un área de estudio ampliamente conocida, sin embargo debe ser examinada más a fondo. A continuación, se muestra una tabla con los resultados obtenidos de varios científicos que han trabajado con nanopartículas de silicio orientados a tratamientos para la conservación de materiales.

Tabla 1. Tratamientos superficiales base nanopartículas de silicio.

\begin{tabular}{|c|c|c|c|c|}
\hline Sustrato & $\begin{array}{c}\text { Ángulo } \\
\text { de } \\
\text { contacto } \\
\text { inicial }\end{array}$ & Durabilidad & $\begin{array}{c}\text { Cambio de } \\
\text { color } \\
\text { significativo }\end{array}$ & Autor \\
\hline Piedra & $138^{\circ}$ & No evaluada & No & (De Ferri, L., et al. 2011) \\
\hline Piedra & $128^{\circ}$ & $\mathrm{Si}$ & No & (Elhaddad, F., et al. 2018) \\
\hline Mortero de Cemento & $119^{\circ}$ & No evaluada & No evaluada & (Ran Li, et al. 2018) \\
\hline Tejas de Arcilla Cocida & $140^{\circ}$ & No & Si & (Carrascosa, L., et al. 2016) \\
\hline Polimérico (PES) & $150^{\circ}$ & No evaluada & Si & (Zhang, X., et al. 2018) \\
\hline
\end{tabular}

Hoy en día la variedad de productos consolidantes, hidrófobos y biocidas, están disponibles comercialmente y se reportan en la literatura científica (Doehne E, 2010). Sin embargo, están plagados de un rendimiento limitado y desventajas como el agrietamiento, la efectividad de corta duración, la 
escasa profundidad de penetración, la baja adherencia, la baja afinidad química, el cambio de coloración y el alto contenido de COV (Hosseini, M., 2018).

En este trabajo, se evaluó un tratamiento superficial con NF sintetizadas en laboratorio, en tejas de arcilla cocida. Así mismo se evaluaron distintos tratamientos comerciales bajo las mismas condiciones. Estas tejas fueron expuestas a un ambiente exterior en una ciudad industrial por 12 meses. La dosificación del tratamiento sintetizado fue de $0.1 \%$ con respecto al volumen de agua necesaria para recubrir la superficie del material. Este porcentaje es considerablemente menor a lo registrado en la literatura y aporta otros beneficios tales como una mayor durabilidad y evita el uso de solventes con compuestos orgánicos volátiles (COV). Los resultados obtenidos mostraron que el producto sintetizado aportó propiedades hidrófobas en la superficie sin cambio significativo en el color del material.

\section{PROCEDIMIENTO}

\subsection{Tejas de Arcilla Cocida}

Se utilizaron tejas de arcilla de fabricación industrial, tipo portuguesa, de color rojo con dimensiones de 41 x $25 \mathrm{~cm}$ con las especificaciones técnicas mostradas en la Tabla 2.

Tabla 2. Especificaciones de las tejas de arcilla.

\begin{tabular}{|l|c|l|c|}
\hline Largo & $7.5 \mathrm{~cm}$ & Ancho & $24 \mathrm{~cm}$ \\
\hline Profundidad & $41 \mathrm{~cm}$ & Material & Arcilla Cocida \\
\hline Color & Rojo & Acabado & Mate \\
\hline Peso & $2.65 \mathrm{~kg}$ & No. de piezas & 1 \\
\hline
\end{tabular}

\subsection{Descripción de los tratamientos superficiales utilizados}

Para la experimentación se utilizaron 6 tejas que fueron divididas en 3 secciones longitudinales cada una. Sobre cada sección se aplicó el tratamiento superficial utilizando productos comerciales y dispersiones elaboradas en laboratorio. También se incluyó una sección referencia sin tratamiento. A continuación, se describen las características de cada sección:

1. Referencia o sección sin tratamiento.

2. Tratamiento comercial A es un impermeabilizante incoloro a base de resina de silicona vehiculizada en solvente, que ofrece protección hidrófuga contra la penetración de agua de lluvia a fachadas o estructuras porosas expuestas a la intemperie, sin cambiar el aspecto original de las superficies.

3. Tratamiento comercial B es repelente al agua, protege hasta varios milímetros de profundidad, elaborado del silicón y aditivos. Protege del agua a muros de concreto prefabricado, piedra, recubiertos con texturizado o estuco. Ideal para materiales pétreos como cantera, granito, terrazo, barro, mármol no pulido, etc. Se obtiene repelencia al secar, la máxima repelencia se da a las 24 horas.

4. Tratamiento comercial $\mathrm{C}$ es un sellador transparente de alto desempeño de silano/siloxano base agua, diseñado para proporcionar protección duradera a muros, bloques de peso ligero y a las unidades de mampostería de concreto estándar.

5. Recubrimiento no comercial de $90 \%$ siloxanos, crea una capa superhidrofóbica.

6. Nanopartículas base silicio funcionalizadas (NF) sintetizadas en laboratorio, protege el material dándole propiedades superhidrofóbicas (muy repelentes al agua). 


\subsection{Aplicación de los tratamientos}

La aplicación de los productos en las muestras de arcilla se realizó con una brocha de espuma de poliuretano (Figura 1), en dos capas. El procedimiento de aplicación utilizado para todos los tratamientos fue el recomendado en las fichas técnicas de los productos comerciales mencionados arriba, y se describe a continuación:

1. Se preparó la superficie lavando los especímenes con un chorro de agua a presión, para posteriormente cepillarlos con un cepillo de cerdas duras, asegurándose que la superficie estuviera limpia de polvo, aceite, manchas, eflorescencias y residuos.

2. La aplicación se realizó por impregnación con una brocha de espuma, cubriendo la superficie dos veces por cara del espécimen para asegurar una mayor penetración. El intervalo de tiempo de aplicación entre capa y capa fue el tiempo total que resultó cuando la primera capa presentó una tonalidad húmeda y posteriormente, la segunda capa se aplicó cuando se observó una tonalidad mate en la primera, esto para evitar el escurrimiento o encharcamiento en la superficie.

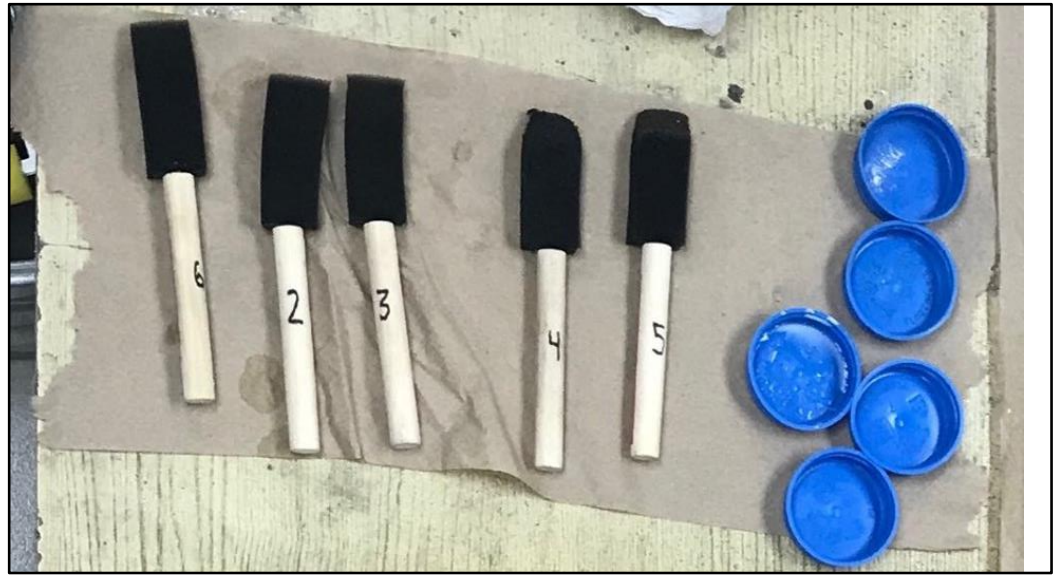

Figura 1. Brochas de espuma de poliuretano para la aplicación de cada tratamiento.

Cada tratamiento se aplicó en 3 secciones verticales de las tejas como se muestra en la figura 4 obteniendo como resultado 18 muestras.

\subsection{Caracterización del ambiente de exposición}

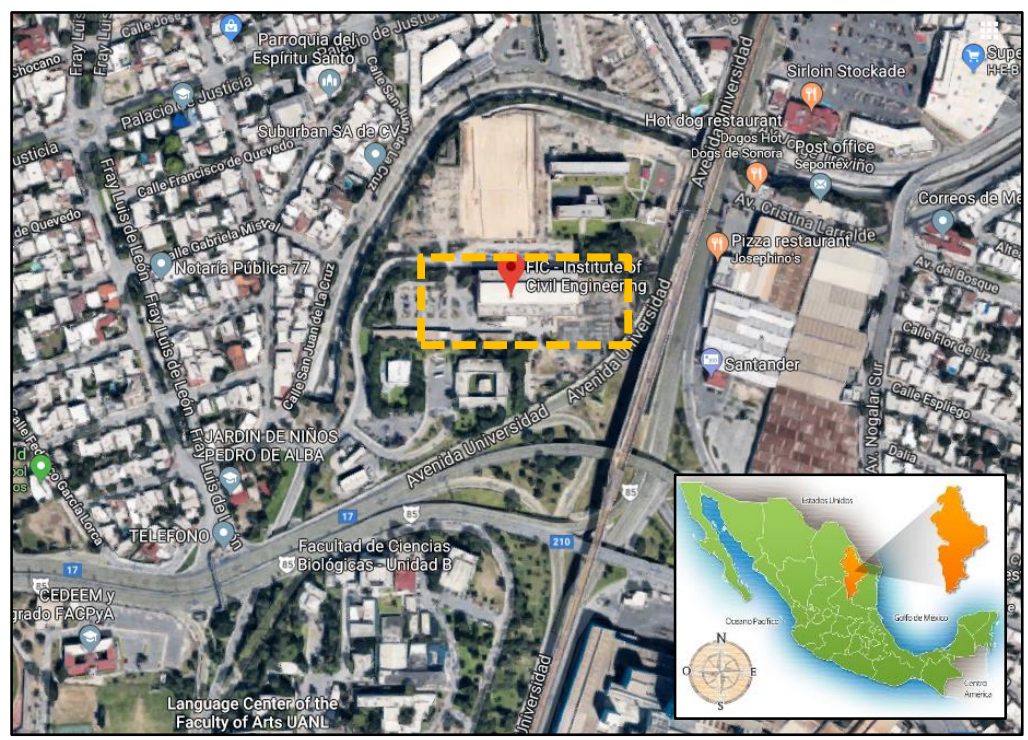

Figura 2. Ambiente de exposición de especímenes. 
Todas las muestras tratadas fueron expuestas al clima de la zona metropolitana de Monterrey N.L., México como se muestra en la figura 2, con el objetivo de evaluar algunas propiedades específicas del recubrimiento en relación con el sustrato. Las muestras tuvieron una ligera inclinación aproximada de $30^{\circ}$ al ser expuestas. De acuerdo con Köppen y Geiger el clima se clasifica como BSh; es decir semiárido cálido.

Las características más importantes son:

- El mes más cálido (con el máximo promedio de temperatura alta) es Julio $\left(34.8^{\circ} \mathrm{C}\right)$.

- El mes con el promedio de temperatura alta más bajo es Enero $\left(20.7^{\circ} \mathrm{C}\right)$.

- El mes con el promedio de temperatura baja más alto es Agosto $\left(22.5^{\circ} \mathrm{C}\right)$.

○ El mes más frío (con el promedio de temperatura baja más bajo) es Enero $\left(8.2^{\circ} \mathrm{C}\right.$ ).

- El mes con la humedad relativa más alta es Octubre $(71 \%)$.

- El mes con la humedad relativa más baja es Marzo $(58 \%)$.

○ El mes más húmedo (con la precipitación más alta) es Septiembre $(150.6 \mathrm{~mm})$.

- El mes más seco (con la precipitación más baja) es Diciembre (14.1mm).

- El mes con el número de días lluviosos más alto es Septiembre (8.2 días).

- Los meses con el número de días lluviosos más bajo son Marzo y Diciembre (3.4 días).

- Los meses con el índice UV más alto son Mayo, Junio, Julio y Agosto (Índice UV 12).

○ Los meses con el índice UV más bajo son Enero y Diciembre (Índice UV 5).

\section{EVALUACIÓN}

\subsection{Apariencia óptica según estándares colorimétricos}

Para evaluar la apariencia óptica se usó el Datacolor marca CHECK que se muestra en la Figura 2, siguiendo el procedimiento e interpretación de resultados según el manual y de acuerdo a la norma ASTM D2244:2016. El instrumento fue previamente calibrado siguiendo el manual y utilizando los aditamentos incluidos. Se procedió a medir tres porciones de cada superficie de la muestra para obtener una indicación de uniformidad. Luego se registró una medición representativa de cada muestra obteniendo los valores de $\mathrm{L}^{*}, \mathrm{a}^{*} \mathrm{y} \mathrm{b}^{*}$, mismos que se proporcionan con el instrumento automáticamente. De esta forma se obtuvo la colorimetría de las 18 muestras.

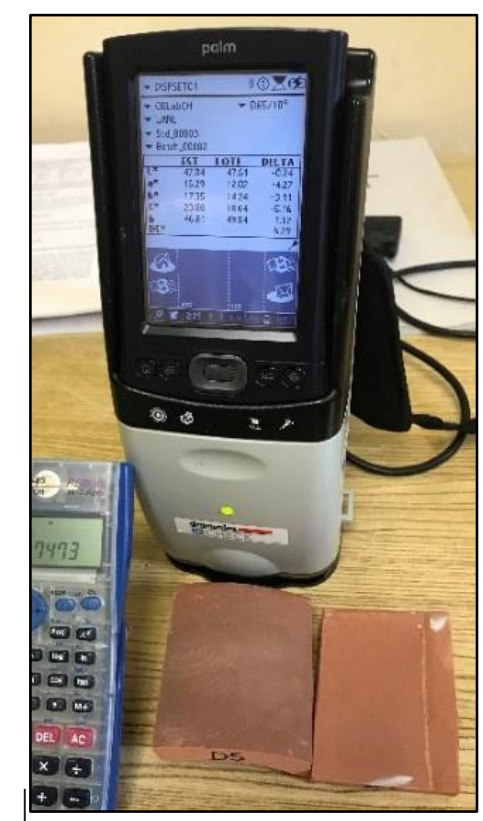

Figura 2. Instrumento Datacolor CHECK. 


\subsection{Medición y evaluación de la hidrofobicidad.}

Para evaluar el desarrollo de la hidrofobicidad en las superficies de las tejas se usó un sistema de análisis de gota consistente en un equipo D5425 Expert Kruss (véase Figura 3) siguiendo la norma ASTM D7334 - 08 para la medición del ángulo de contacto. Las muestras se midieron a $23 \pm 2{ }^{\circ} \mathrm{C}$ y con una $\mathrm{HR} \geq 50 \%$. Se usó un volumen de gota de $6 \mu \mathrm{L}$. Se realizaron dos mediciones de ángulo (una en cada punto de caída) de cada una de las tres gotas que fueron depositadas sobre la muestra. En los ángulos de contacto con mediciones significativamente diferentes en dos bordes, se eliminaron y la prueba se repitió en dichos casos. El ángulo de contacto para el espécimen fue el promedio de los seis ángulos medidos. Los ángulos de contacto con el agua deben medirse rápidamente por lo que se midieron en un periodo no mayor de 30 s después de depositarse la gota.

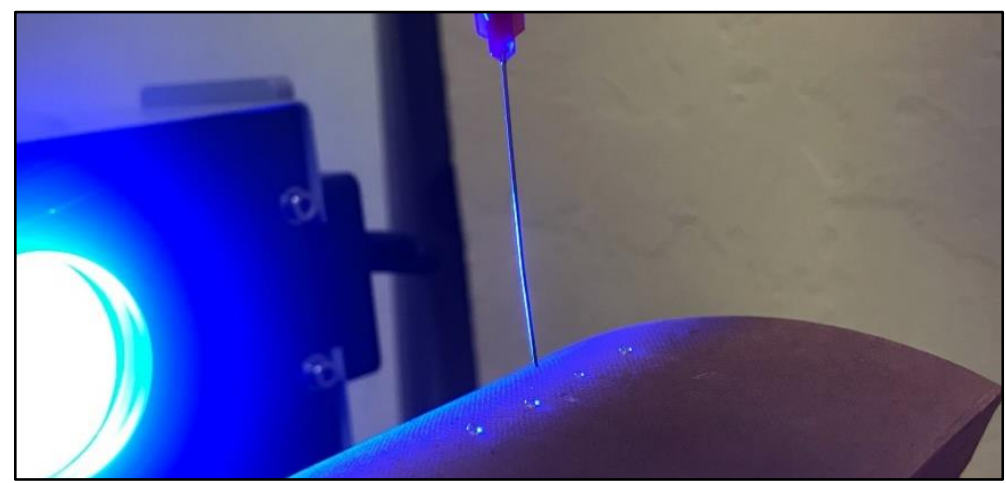

Figura 3. Medición del ángulo de contacto en teja de arcilla cocida tratada.

\section{RESULTADOS Y DISCUSIÓN}

\subsection{Apariencia óptica según estándares colorimétricos}

En la Figura 4 se presenta una imagen, de las tejas de arcilla, obtenida después de 12 meses de exposición ambiental (azotea del IIC). Los números indican las diferentes secciones con los tratamientos descritos en el Apartado 2.2.

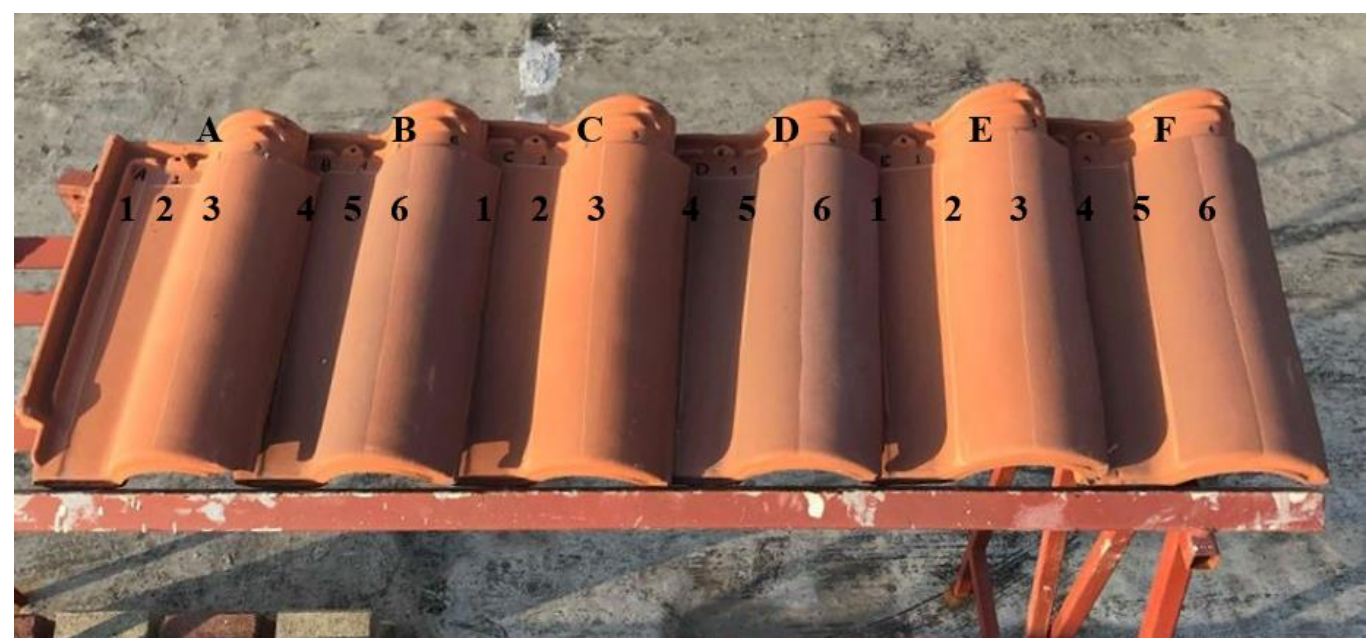

Figura 4. Imagen de los especímenes a 12 meses después de la aplicación de los tratamientos: (1) sección de referencia sin tratar; (2) sección tratada con el tratamiento comercial A; (3) sección tratada con el tratamiento comercial B; (4) sección tratada con el tratamiento comercial C; (5) sección tratada con $90 \%$ siloxanos y (6) sección tratada con NF. 
Como se puede constatar, la superficie de las tejas con tratamientos comerciales (\#2, \#3 y \#4) muestran un cambio de coloración en comparación con la referencia (\#1). Es de resaltar que hasta el momento, las muestras tratadas con NF (\#6) visualmente presentaron un ligero cambio de aspecto.

Las mediciones colorimétricas fueron realizadas de forma comparativa, en muestras tratadas y no tratadas. La diferencia de color $\left(\Delta E^{*}\right)$ se determinó con la ecuación 1 y se reportan en la Tabla 3.

$$
\Delta \boldsymbol{E}^{*}=\sqrt{\Delta \boldsymbol{L}^{* 2}+\Delta \boldsymbol{a}^{* 2}+\Delta \boldsymbol{b}^{* 2}}
$$

Donde $L^{*}, a^{*}, y b^{*}$ son las coordenadas estándares de la escala CIELab color space 1976.

Tabla 3. Resultados de colorimetría obtenidos a 12 meses de exposición.

\begin{tabular}{|l|c|}
\hline Tratamiento & $\Delta \boldsymbol{E}^{*}$ \\
\hline A ó \#2 & 4.3 \\
\hline NF ó \#6 & 4.7 \\
\hline B ó \#3 & 5.1 \\
\hline Co o4 & 5.5 \\
\hline 90\% Siloxanos ó \#5 & 7.8 \\
\hline
\end{tabular}

La diferencia de color $\left(\Delta E^{*}\right)$ fue mayor en el tratamiento de siloxanos que visualmente oscureció significativamente la muestra, seguido por dos de los productos comerciales. El tratamiento sintetizado obtuvo un cambio de coloración mínimo, como consecuencia del ligero blanqueamiento (valores positivos para $\Delta \mathrm{L} *$ ) inducido por el tratamiento. Sin embargo, estos cambios podrían ser aceptables para estos tipos de materiales de la construcción. Cabe mencionar que la diferencia de color $\left(\Delta E^{*}\right)$ entre 2 y 3.5 es la que comúnmente percibe por el ojo humano.

\subsection{Comportamiento de la hidrofobicidad}

Los tratamientos aplicados deben producir hidrofobicidad en la muestra de arcilla cocida tratada. El valor inicial del ángulo de contacto no se obtuvo debido a que no se contaba con el equipo, sin embargo, se confirmó la hidrofobicidad aplicando agua en la muestra con una pipeta de laboratorio. Como se muestra en la Figura 5, en todas las muestras tratadas se desarrolló una superficie hidrofóbica caracterizada por el comportamiento del agua, la cual no mostró adherencia o absorción en dicha superficie.

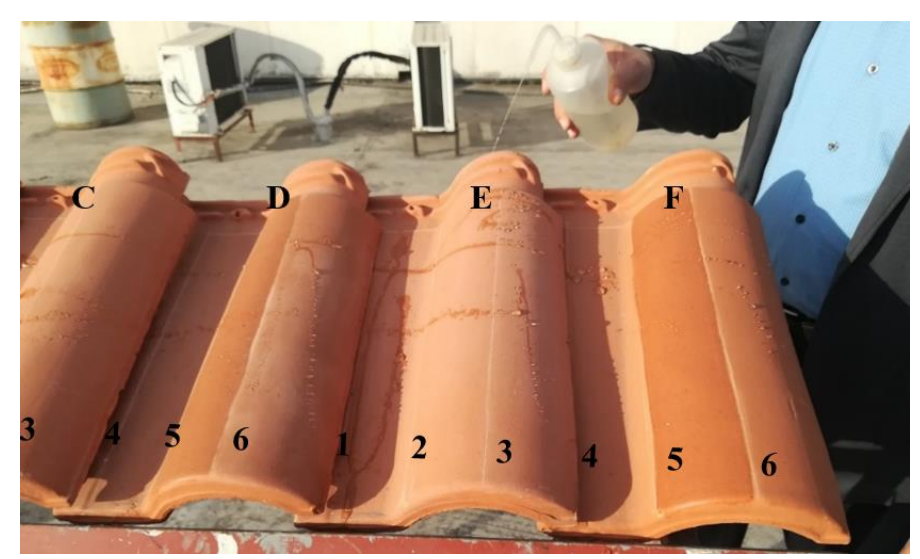

Figura 5. Especímenes después de la aplicación del tratamiento (22/01/2018). (1) sección de referencia sin tratar; (2) sección tratada con el tratamiento comercial A; (3) sección tratada con el tratamiento comercial B; (4) sección tratada con el tratamiento comercial C; (5) sección tratada con $90 \%$ siloxanos y (6) sección tratada con NF. 
Los resultados de la medición del ángulo de contacto de las muestras después de 12 meses de exposición se muestran en la Fig. 6.

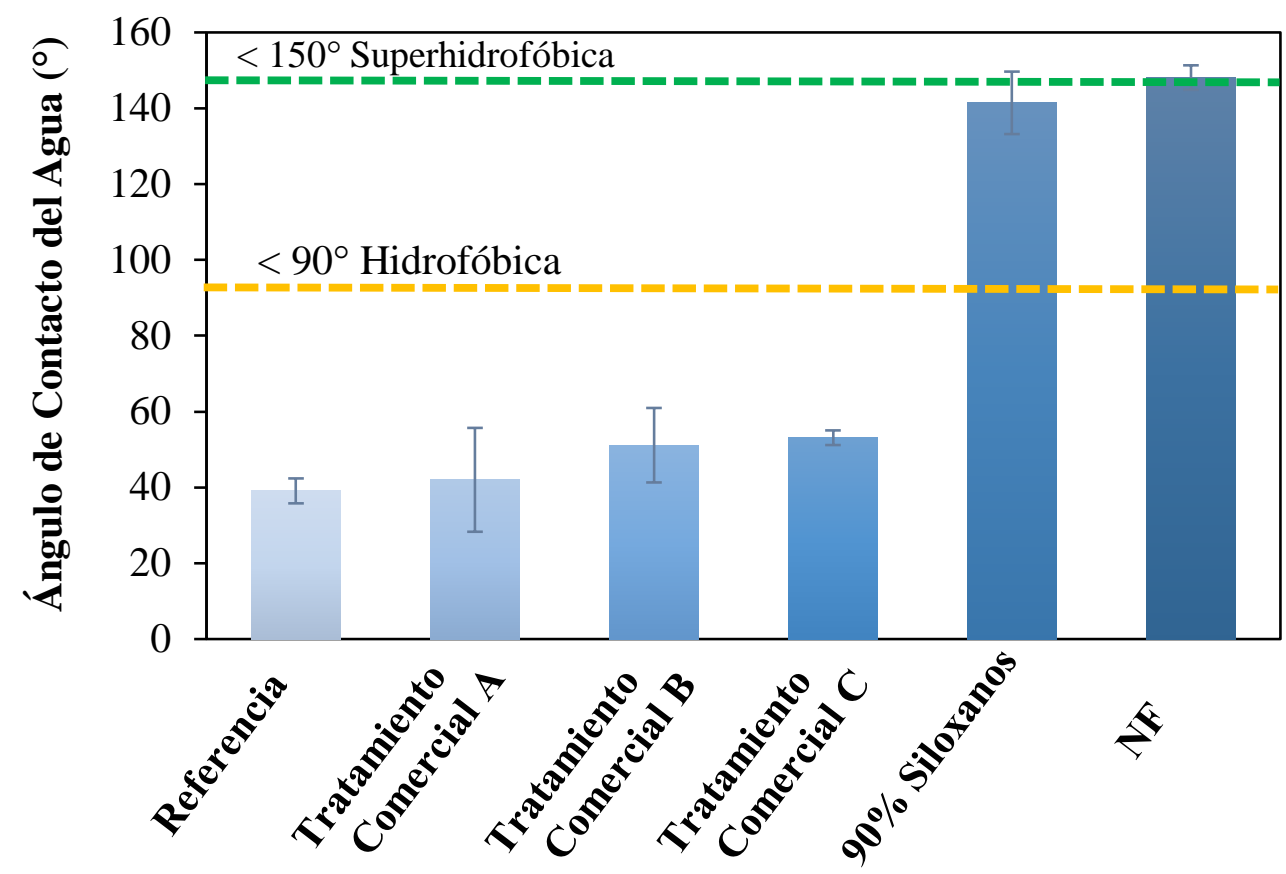

Figura 6. Ángulo de contacto en muestras de arcilla cocida con diferentes tratamientos.

Como se puede constatar de la figura, el ángulo de contacto disminuyó sensiblemente en los tratamientos comerciales comparados con los tratamientos en donde se utilizó NF y siloxanos. La disminución de la hidrofobicidad puede atribuirse a la degradación de los recubrimientos debido a la exposición de radiaciones U.V., a la lluvia y a los compuestos contaminantes presentes en el ambiente industrial de Monterrey. La pérdida de hidrofobicidad expone al material a los diversos tipos de deterioro promovidos por el agua, dejando al material indefenso ante los agentes químicos agresivos presentes en la atmósfera. Adicionalmente, se puede generar humedad en el material promoviendo el desarrollo de biomasa. Los resultados positivos de la diferencia de color se desprecian al haberse perdido el efecto que se buscaba con los productos comerciales, los cuales eran "sellar, impermeabilizar y repeler el agua" de diversos materiales de construcción.

\section{CONCLUSIÓN}

El uso de NF en tratamientos superficiales muestra resultados muy positivos. Las NF proveen al material propiedades superhidrofóbicas aún después de un año de su aplicación, esto evidenciado por su elevado ángulo de contacto de $150^{\circ}$. Adicionalmente, este recubrimiento no cambia significativamente el color del material, por lo que el uso de las nanopartículas puede proporcionar un resultado integral. Las propiedades hidrófobas protegen al material del agua de lluvia y contaminantes, lo que podría promover el efecto autolimpiante, como consecuencia obteniendo superficies antihumedad, antiadhesión, resistentes a las manchas y a la proliferación de organismos. El uso potencial de NF como tratamiento superficial para la protección de nuevas edificaciones, así como monumentos de interés histórico es una idea prometedora, sin embargo, deben estudiarse diversos factores tales como su duración, comportamiento y efectos secundarios a largo plazo y bajo condiciones de exposición a microorganismos, por ejemplo. 


\section{AGRADECIMIENTOS}

Los autores agradecen al fondo SEP-CONACYT por el apoyo financiero otorgado al proyecto CB2016-01/285453. M. Neri-Alvarez, agradece la beca CVU \#872560 para la realización de sus estudios de posgrado.

\section{REFERENCIAS}

American Concrete Institute (1990), ACI 116R-90, Cement and Concrete Terminology. Michigan. ASTM International. (2016). ASTM D2244-16 Standard Practice for Calculation of Color Tolerances and Color Differences from Instrumentally Measured Color Coordinates. https://doi.org/10.1520/D2244-16

BASF Corporation (2014), MasterProtect ${ }^{\circledR} H$ 185: Sellador repelente al agua de alto desempeño base agua con mezcla patentada de silano/siloxano para uso en bloques de peso ligero. Hoja Técnica. Master Builders Solutions. Disponible en : https://assets.master-builderssolutions.basf.com/shared\%20documents/eb\%20construction\%20chemcials\%20-

\%20us/construction\%20systems/data\%20guides/masterprotect/spanish/basf-masterprotect-h-185-tdssp.pdf

Bhushan, B., Jung, Y. C., \& Nosonovsky, M. (2010). Lotus Effect: Surfaces with Roughness-Induced Superhydrophobicity, Self-Cleaning, and Low Adhesion. Springer Handbook of Nanotechnology, 1437 1524. doi: https://doi.org/10.1007/978-3-642-02525-9_42

Boostani, H., \& Modirrousta, S. (2016). Review of Nanocoatings for Building Application. Procedia Engineering, 145, 1541-1548. doi: https://doi.org/10.1016/j.proeng.2016.04.194

De Ferri, L., Lottici, P. P., Lorenzi, A., Montenero, A., \& Salvioli-Mariani, E. (2011). Study of silica nanoparticles - polysiloxane hydrophobic treatments for stone-based monument protection. Journal of Cultural Heritage, 12(4), 356-363. https://doi.org/10.1016/j.culher.2011.02.006

Elhaddad, F., Carrascosa, L. A. M., \& Mosquera, M. J. (2018). Long-term effectiveness, under a coastal environment, of a novel conservation nanomaterial applied on sandstone from a Roman archaeological site. Journal of Cultural Heritage, (2017). https://doi.org/10.1016/j.culher.2018.04.013

Hosseini, M., \& Karapanagiotis, I. (2018). Advanced Materials for the Conservation of Stone. https://doi.org/10.1007/978-3-319-72260-3

IMPAC (2014), Repelente de agua a base de silicón para fachadas, muros, y materiales pétreos. Disponible en: http://www.impac.com.mx/fichas/IMPACClear.pdf

Instituto Nacional de Estadística y Geografía (2018) Anuario estadístico y geográfico de Nuevo León 2018, México: INEGI.

Liu, Y., \& Liu, J. (2016). Synthesis of TEOS / PDMS-OH / CTAB composite coating material as a new stone consolidant formulation. Construction and Building Materials, 122, 90-94. https://doi.org/10.1016/j.conbuildmat.2016.06.069

Shaquihuanga-Ayala, D., (2014). Evaluación del estado actual de los muros de albañilería confinada en las viviendas del sector Fila Alta -Jaén, Universidad Nacional de Cajamarca.

SIKA (2013), Sikaguard® 70: Impermeabilizante incoloro para fachadas y muros exteriores, a base de siliconas vehiculizadas en solvente, Hoja técnica de producto, Edición 09/07/2013, $\mathrm{N}^{\circ}$ de

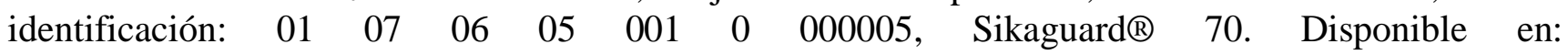
https://mex.sika.com/content/dam/dms/ar01/8/Sikaguard\%2070.pdf

Verheye, W. H. (2009). Encyclopedia of land use, land cover and soil sciences. Oxford: EOLSS.

Yu Media Group. (n.d.). Monterrey, México - Información detallada del clima y previsión meteorológica mensual. Retrieved from https://www.weather-mx.com/es/mexico/monterrey-clima Luis A. M. Carrascosa, Dario S. Facio, Maria J. Mosquera. (2016). Producing superhydrophobic roof tiles. Nanotechnology 27095604 (13pp) doi: https://doi.org/10.1088/0957-4484/27/9/095604 
Zhang, X., Liu, Z., Zhang, X., Li, Y., Wang, H., Wang, J., \& Zhu, Y. (2018). High-adhesive superhydrophobic litchi-like coatings fabricated by in-situ growth of nano-silica on polyethersulfone surface. Chemical Engineering Journal, 343(March), 699-707. https://doi.org/10.1016/j.cej.2018.03.012

Zhu, Y., Chen, L., Zhang, C., \& Guan, Z. (2018). Preparation of hydrophobic antireflective SiO2 coating with deposition of PDMS from water-based SiO2-PEG sol. Applied Surface Science, 457, $522-$ 528. doi: https://doi.org/10.1016/j.apsusc.2018.06.177 